\title{
Costumbres lúdicas en España: el juego de bolos en la etapa final del camino de Santiago
}

\author{
José Eugenio Rodríguez Fernández* \\ José María Pazos Couto** \\ José Palacios Aguilar***
}

\begin{abstract}
Resumen: El estudio buscó identificar y analizar las prácticas lúdicas de los habitantes del noroeste de España y la relación que se establece con las diferentes vías marítimo-terrestres del Camino de Santiago que penetran en Galicia. Los datos se obtuvieron a través de una metodología cualitativa, utilizando la entrevista semiestructurada y la revisión bibliográfica. Se constató un gran paralelismo entre las vías de entrada del Camino de Santiago en Galicia y los municipios donde sigue vigente el juego de bolos, verificando las influencias de los peregrinos en su camino a Santiago con fines espirituales, trasladando sus costumbres y tradiciones.

Palabras clave: Cultura. Juego y implementos de juego. Actividades recreativas.
\end{abstract}

\section{INTRODUCCIÓN}

La riqueza cultural de un determinado pueblo o sociedad puede ser modelada de múltiples formas y en este proceso influyen una gran diversidad de aspectos, entre los que se encuentra una forma particular de ocio: los juegos populares y tradicionales, que constituyen el reflejo social y que revela los rasgos de identidad y culturales de una sociedad de ocio tradicional (VIGNE, 2011). Los juegos tradicionales, además de ocupar un lugar destacado en las prácticas de ocio y tiempo libre, constituyen un vehículo perfecto para transmitir los rasgos de identidad de las personas $y$, sobre todo, los valores de un pueblo, de una sociedad que forjó a fuego lento la historia de su gente a través de ciertas prácticas que,

\footnotetext{
*Universidad de Santiago de Compostela. España. E-mail: uxiorf@hotmail.com

**Universidad de Vigo. Pontevedra. España. E-mail: chema3@gmail.com

***Facultad de Ciencias del Deporte y la Educación Física. Universidad de A Coruña. España.

E-mail: jose.palacios@udc.es
} 
por costumbre, se desarrollaron de generación en generación hasta nuestros días.

Dentro del amplísimo abanico de juegos y deportes populares y tradicionales que nos podemos encontrar en el conjunto del territorio español hay uno que, como afirma Lavega (1996), destaca por su singularidad, por su propia condición de juego que sobrevivió al paso del tiempo y, como afirma Moreno (1992), por su extraordinaria presencia en las distintas comunidades autónomas. Se trata de un juego secular, los bolos, una actividad física (que exige sobre todo la implicación de la capacidad física de la fuerza y la habilidad de precisión), un juego muy antigüo y muy extendido en todas las culturas (RUIZ, 2000) (en el que la principal finalidad es puro divertimiento y recreación, con reglas, jugadores e instalaciones flexibles, adaptados a las necesidades de cada momento) y, según que modalidades, un deporte (que supone una evolución del juego, sometido a un reglamento específico, en una instalación concreta y bajo la supervisión de un árbitro, vinculado en este caso principalmente a la actividad federada); se trata de una actividad con un marcado componente histórico pero que, con el paso del tiempo, ha ido perdiendo protagonismo en favor de los denominados deportes modernos o juegos deportivos institucionalizados (LAVEGA, 2000a; PARLEBÁS, 2003).

El juego de bolos, uno de los más practicados en todo el mundo en sus diferentes variantes y modalidades (RUIZ, 2000), tiene una presencia diferente en los diferentes pueblos españoles, concentrándose una mayor actividad en la zona noroccidental de la península, destacando la actividad generada en la zona de País Vasco, Cantabria, Asturias, León y Galicia. Los habitantes de estas zonas han luchado para que sus costumbres y tradiciones sobrevivan, utilizando para ello aspectos como la literatura, el arte, la arquitectura, el lenguaje, la educación o las formas de entretenimiento (COOMBS, 1985); pero, sobre todo, creyeron que podían contribuir a la conservación de las tradiciones propias de cada pueblo, tradiciones que reflejan la historia de la gente, de sus antepasados, a través del juego de bolos. 
Galicia posee un importante bagaje cultural en gran parte determinado por las costumbres de los habitantes de esta region del noroeste de España. Los juegos populares y tradicionales son parte importante de la historia de los pueblos gallegos y, en especial, el juego de bolos. Galicia fue además el centro de uno de los movimientos religiosos y culturales más grandes desde la Edad Media, el Camino de Santiago.

Con origen en el siglo IX, el Camino de Santiago toma fuerza como ejemplo de consolidación del nuevo reino astur, único reducto fiel en la Península Ibérica contra el expansionismo del Islam. Con la elevación a mito del Apóstol Santiago y con el beneplácito de la monarquía e iglesia católica, se desarrolla a partir de entonces un intenso proceso peregrinatorio desde todos los rincones de Europa hasta Compostela, surgiendo una serie de rutas que van más allá de las motivaciones simplemente religiosas (SANTOS, 2006).

Desde sus inicios, el Camino de Santiago se constituyó como una incesante fuente de espiritualidad, de trasmisión de la cultura y las tradiciones, permitiendo la entrada de nuevos gustos provenientes de otros países como pudieron ser los artísticos (con su influjo en numerosísimos edificios religiosos -más de 1.800y construcciones civiles de interés histórico) o los literarios (con personajes como los juglares), sin olvidar que fue la conexión entre la Península y el continente europeo, situándose junto a Jerusalén y Roma como lugar sagrado que todo cristiano aspiraba a visitar al menos una vez en la vida, siendo el símbolo por excelencia del cristianismo español (ECHEVARRÍA, 2008).

Pero la importancia del Camino de Santiago no radica sólo en las obras artísticas y los lazos espirituales que nos ha dejado, sinó también en los intercambios culturales y económicos establecidos en esta red de peregrinación. Para darle mayor personalidad y transcendencia al Camino, se creó todo un culto de santos menores a lo largo de la peregrinación, se establecieron nuevos asentamientos de emigrantes francos, se revitalizó la economía a través del comercio -hospedaje, alimentación, artesanía, oficios

Movimento, Porto Alegre, v. 20, n. 4, p. 1397-1421, out./dez. de 2014. 
diversos-, resultando ser un poderoso factor de dinamización económica (SANTOS, 2006).

El Camino de Santiago, declarado Patrimonio de la Humanidad por la UNESCO en 1993, ha significado en la historia europea el primer elemento vertebrador del viejo continente. Jugó un papel fundamental en el fenómeno de intercambios culturales entre la Península Ibérica y el resto de Europa, propiciando un desarrollo cultural y económico por las zonas que discurría, donde los peregrinos, españoles o extranjeros, traían con ellos sus tradiciones y gustos, poniendo en contacto a éstos con las nuevas tendencias y modas ultrapirenaicas, hecho que perdura hoy en día.

El objeto de este trabajo es conocer la realidad de la práctica del juego de bolos ${ }^{1}$ en diferentes municipios gallegos y su vinculación con las prácticas antiguas de los peregrinos que se dirigían a Compostela a venerar las reliquias del apóstol Santiago.

La bibliografía, documentación y, en general, los datos históricos sobre o xogo dos birlos en Galicia (COSTAS; RODRÍGUEZ, 2000; BERMÚDEZ, 2007) son muy escasos, siendo este un fenómeno con poco tratamiento en el campo de la investigación en España y, en relación a los bolos y en Galicia, pasaría por ser un trabajo inédito en este sentido.

\section{EL JUEGO Y LAS TRADICIONES}

El juego, como una actividad inherente al ser humano y apreciado como una necesidad vital (VEIGA, 1998), es un término

\footnotetext{
${ }^{1}$ El juego de bolos en Galicia destaca por ser una modalidad de pasabolo, donde el objetivo pasa por derribar y desplazar lo más lejos posible un determinado número de bolos (de 6 a 18 bolos, variable según la zona geográfica de juego). Se juega al aire libre en campos de bolos de forma rectancular, donde los bolos pueden alcanzar la distancia de hasta 80 metros. Estas características lo diferencian de otras modalidades que se practican en varios lugares del mundo, como pueden ser el juego de boliche y bolão de Brasil (donde el objetivo consiste en derribar 9 bolos y que se juega en pistas de madera a cubierto), semejante al bowling americano y diferente de otras modalidades como la bocha brasileña (en España denominada petanca), donde el objetivo es situar esferas lo más cerca posible a un objeto lanzado previamente (bochín) y que, a pesar de que exigen la fuerza y precisión del juego de bolos, el objetivo del juego difiere considerablemente.
} 
estudiado por una gran variedad de autores dada su significación polisémica (EXPÓSITO, 2006), sus raíces etimológicas (HUIZINGA, 1972) y conceptualización (CAGIGAL, 1996).

Eljuego posee unas determinadas características (BAUTISTA, 2002; MELERO, 2009) que lo convierten por encima de todo en una actividad placentera y socializadora, determinando una gran variedad de funciones y posibilidades que se pueden adquirir con su práctica (BARROSO, 2003; LAVEGA, 2006) y englobando una serie de valores que posibilitan el desarrollo evolutivo de la persona en diferentes ámbitos: cultural, social, educativo, afectivo o físico (ROMERO, 2007; LARA, 2011).

Dentro del panorama de los juegos y los deportes hay un tipo particular de ellos, quizás los más antiguos, transmitidos ${ }^{2}$ de generación en generación y que poseen un marcado carácter cultural desde el punto de vista antropológico (MORENO, 1992). Son los juegos y deportes populares y tradicionales, que poseen un indudable valor cultural, que refleja la cultura que lo rodea y la sociedad en la que está inmerso (LAVEGA, 1994), aflorando aspectos como la etnopracticidad (HUIZINGA, 1972), etnoludismo (LAVEGA, 1997) ${ }^{3}$, etnomotricidad (PARLEBÁS, 2003), enculturación (NAVARRO, 2003), aculturación (DONCEL, 2010) o sincretismo (HERNÁNDEZ, 2007).

Desde siempre el hombre jugó y desarrolló actividades lúdicas como forma de expresión, de ocio, de esparcimiento (BARROSO, 2003). El pueblo gallego fue capaz de crear

\footnotetext{
${ }^{2}$ La transmisión de las tradiciones, representativas de comarcas o lugares con un carácter cultural propio, se producen debido a su particularidad, siendo reflejo de la idiosincrasia cultural de esos propios lugares. Referidos a los hábitos o costumbres, las tradiciones se ven fortalecidas por las rutinas, por una actividad cotidiana que refleja la forma de proceder de la gente, encontrando en la familia el círculo de transmisión más habitual. En este caso, la transmisión se produce de abuelos a padres y de padres a hijos. En el caso del juego de bolos, los jóvenes que se iniciaban en el juego eran examinados por el colectivo en base a las actitudes y habilidades de sus generaciones anteriores, asumiendo a partir de ese momento la responsabilidad de continuar con la tradición familiar que, de forma más reciente, había recaído en su padre.

${ }^{3}$ LAVEGA, Pere. Aplicación educativa/recreativa del juego a partir de sus distintos niveles de organización. Comunicación presentada en el 20th World Play Conference "Play And Society". Lisboa: ICCP Facultad Motricidad Humana. 1997.
}

Movimento, Porto Alegre, v. 20, n. 4, p. 1397-1421, out./dez. de 2014. 
infinidad de maneras de dar respuesta a sus necesidades lúdicas, al tiempo que también tuvo la capacidad de transmitir esas creaciones a las nuevas generaciones.

En Galicia, los juegos nacieron como las creencias, las costumbres (VEGA, 1990) e incluso la forma de poblamiento, ligados a la forma de producción de una sociedad esencialmente agraria, que los convertía en una evolución del trabajo (VEIGA, 1998), de ahí las diferencias entre los juegos del interior y de la costa gallega (CORTIZAS, 2001), entre las zonas rurales y urbanas (ROMANÍ, 1979) o mismo entre niñas/mujeres o niños/hombres (LIÑARES, 2007).

La práctica profusa de los juegos y deportes populares y tradicionales en Galicia tiene su punto de inflexión a mediados del siglo XX (PÉREZ; TABERNERO, 1997), achacable a la irrupción y popularización progresiva de los juegos deportivos, los deportes de ámbito nacional y a la introducción de nuevos hábitos de consumo en el mundo del ocio: televisión, ordenador o juguetes comerciales, entre otros (RODRÍGUEZ, 2013).

\section{EL JUEGo de bolos Como REFERENTE CULTURAL}

Es muy difícil de precisar si el juego de bolos es el más antiguo de los juegos (LÓPEZ, 2009), pero existen argumentos suficientes que señalan que el hombre primitivo debió disfrutar de una versión de este juego (BRAUN, 1984) y que existen referencias sueltas de la práctica del juego de bolos desde Malasia, Turquía, el Egipto de los faraones, pasando por la Grecia clásica, el Imperio Romano, la América precolombina, la edad media hasta la edad moderna (RUIZ, 2000).

Además de las influencias de las culturas romana y visigodas durante los primeros años de la era cristiana, o de la cultura celta o pre-celta (ARANZADI, $1917^{4}$ citado por LAVEGA, 2000a), las peregrinaciones a Compostela por el Camino de Santiago motivó

\footnotetext{
${ }^{4}$ ARANZADI, T. de; HOYOS SÁINZ, Y L. de. Etnografía: sus bases, sus métodos y aplicaciones a España. Villanueva: Biblioteca Corona, 1917.
}

Movimento, Porto Alegre, v. 20, n. 4, p. 1397-1421, out./dez. de 2014. 
la asimilación de costumbres extranjeras, sobre todo francesas y germanas, estableciéndose por los pueblos, villas y ciudades del norte desde Navarra hasta Santiago de Compostela.

Los peregrinos convirtieron el camino hasta el sepulcro en algo más que una vía de tránsito. Se fundaron posadas, se levantaron puentes, se construyeron iglesias, monasterios y abadías y, por supuesto se formaron núcleos de población que constituyen aún hoy en día un enorme legado artístico y cultural (BRAUN, 1984). Con ellos se introdujeron sus juegos y, entre ellos, seguramente el antiguo juego de bolos alemán del Kegel o alguna de sus variantes, que con el tiempo evolucionaría en nuestro país (FERNÁNDEZ, 1978; BRAUN, 1984).

La pluralidad y diversidad del juego de bolos es tal que, para estudiar su itinerario cultural desde su posible origen, es necesario hacerlo desde una perspectiva sociocultural (porque todo juego popular y tradicional responde a un hecho social y cultural que de algún modo refleja la forma de ser de su gente, donde sus protagonistas al crear un juego establecen un conjunto de relaciones entre ellos que al jugar ponen de manifiesto unha estructura profunda, compleja, llena de riesgos del universo social y cultural al que pertenecen) y desde una perspectiva contextual (ya que en cada contexto, sea cual sea el lugar y la fecha, los juegos populares y tradicionales se manifiestan en condiciones peculiares, circunstancia que los hacen verdaderamente significativos y representativos en ese contorno espacial y temporal en el que se practican (LAVEGA, 2000b).

El juego de bolos se manifiesta de este modo como un referente cultural del lugar donde se practica, cada uno con sus propias características y su propia historia.

\section{REVISIÓN BIBLIOGRÁFICA}

El punto de partida del estudio se inicia con una extensa revisión bibliográfica con el objetivo de conocer el estado de la cuestión, realizando un análisis de los estudios realizados en los

Movimento, Porto Alegre, v. 20, n. 4, p. 1397-1421, out./dez. de 2014. 
últimos años sobre esta temática, recurriendo a las bases de datos de Teseo, Isbn, Iacobus, Dialnet, Sport Discus y Wok mediante la búsqueda de palabras clave clasificadas por niveles para buscar una mayor exhaustividad en la revisión.

En una primera búsqueda nos encontramos con 4.515 documentos, seleccionando 389 después de la revisión. Para el análisis de la información recopilada se clasificaron los documentos encontrados según su temática: bolos, aspectos sociales y culturales, aspectos educativos y otros aspectos (psicológicos, deporte, catalogación...), reseñando que la mayor parte de los estudios encontrados hacían referencia a aspectos socioculturares y educativos de los juegos populares y tradiconales en general y que se encontraron pocos estudios publicados en relación al juego de bolos, de las que hay que destacar tres tesis doctorales (LAVEGA, 1996; RUIZ, 2000; RODRÍGUEZ, 2013).

\section{Material y método}

\subsection{DiseÑo}

Para aclarar las hipótesis de este estudio optamos por la investigación cualitativa como estrategia metodológica (CANALES, 2006), encuadrada bajo un paradigma descriptivo e interpretativo (CORBETTA, 2007) y donde la elaboración de la teoría y la investigación empírica se produce de forma simultánea (PAZOS, 2010).

Utilizamos esta metodología para este caso concreto porque es la más adecuada para estudiar y conocer las prácticas de los participantes y las interrelaciones con un determinado contexto (FLICK, 2007); porque es la más empleada para investigaciones de tipo social y recurre prioritariamente a diseños metodológicos de carácter cualitativo para la recogida de datos; porque se interesa por lo que hace la gente, cómo se comporta y cómo interactúa con los demás; porque se apoya en la convicción de que las 
tradiciones, roles, valores y normas del ambiente en que se vive se van interiorizando poco a poco y generan determinados estilos que pueden explicar la conducta de las personas de forma adecuada $\mathrm{y}$, sobre todo, porque le da mucha importancia a la cultura y, de este modo, entiende los fenómenos que se van a investigar en términos socioculturales, dedicándose a la observación y comprensión de aspectos de una cultura o grupo social determinado, destacando las costumbres o los modos de vida (TÓJAR, 2006).

\subsection{MUESTRA}

La muestra estuvo formada por 50 entrevistados en tres grupos con los siguientes perfiles: 45 personas (técnicos deportivos/ culturales municipales y políticos locales), 1 persona (presidente de la Federación Gallega de Bolos) y 4 personas (directivos/jugadores de clubs de bolos federados - provincias de Pontevedra, Lugo y Orense- y sin federar - provincia de A Coruña-).

\subsection{INSTRUMENTO}

La entrevista fue la principal herramienta metodológica empleada para la obtención de datos (CANALES, 2006; TÓJAR, 2006), seleccionando aquellos informantes que por sus características nos podían aproximar más al núcleo de acción, tal y como requiere este tipo de investigaciones cualitativas (RUIZ; CABRERA, 2004). Se trata de una entrevista semiestructurada, realizada preferentemente por teléfono y por un único investigador, con formación y experiencia en este tipo herramientas cualitativas. Los ítems y la entrevista en su conjunto fue validada por dos expertos universitarios (profesores) con experiencia en este tipo de investigaciones.

\subsection{Procedimiento}

El desarrollo del estudio se realizó en tres partes. La primera en base a la obtención de datos de la revisión bibliográfica y, 
sobre todo, del análisis del estudio de investigación de la realidad de los juegos y deportes populares y tradicionales en Galicia que la Fundación Deporte Galego y la Dirección Xeral para o Deporte de la Xunta de Galicia elaboraron en el año 2009 (no publicado) con el objetivo de conocer en que municipios se estaban promoviendo este tipo de actividades referente a modalidades de juego, entidades implicadas, equipamientos, instalaciones e incluso personal. La segunda parte en base a la entrevista al presidente de la Federación Gallega de Bolos, para conocer el panorama general del juego de bolos en la comunidad gallega. La tercera parte en base a las entrevistas realizadas a técnicos deportivos/culturales, políticos locales, directivos y jugadores de bolos de las localidades en las que se registraba actividad bolística.

Para la elaboración de las diferentes partes de este estudio de investigación se siguió un método de trabajo basado en la elaboración de mapas mentales (BUZÁN, 2004; ONTORIA; GÓMEZ; LUQUE, 2006), con el objetivo de guiar el trabajo de una forma estructurada y organizada (MUNOZ, 2009), empleando herramientas informáticas como el Xmind o Freemind.

Una vez extraídos los datos de las entrevistas se procedió con el análisis e interpretación de la información recogida, realizando un tratamiento manual de los datos con el convencimiento de realizar un análisis eficaz y controlado y en el que los resultados se corresponden fielmente con la realidad estudiada.

\section{Resultados}

Los peregrinos que se dirigían a Santiago de Compostela podían hacerlo por 7 vías diferentes (ver figura 1) que atravesaban las cuatro provincias gallegas hasta llegar a la Catedral de Santiago: por Lugo (Camino del Norte o de la Costa, Camino Primitivo y Camino Francés), por Ourense (Camino de la Plata), por Pontevedra (Camino Portugués), por A Coruña (Camino Inglés y Ruta Fluvial) (POMBO, 2011).

Movimento, Porto Alegre, v. 20, n. 4, p. 1397-1421, out./dez. de 2014. 
Costumbres lúdicas en España: el juego de bolos ...

Figura 1. Mapa general de los Caminos de Santiago a su paso por Galicia.

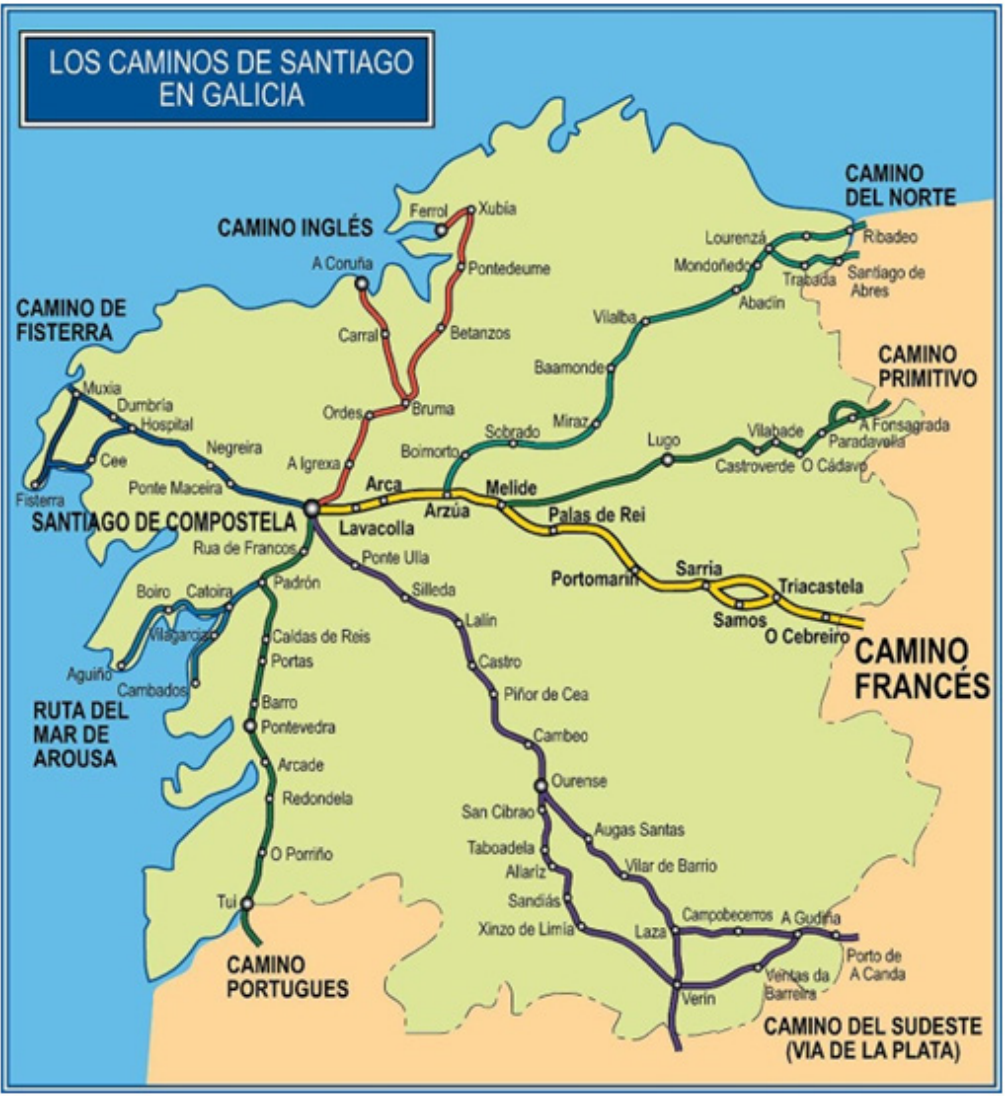

Fuente: www.espormadrid.es/2010/10/los-caminos-de-santiago-en-galicia.html

\subsection{EL JUEGO DE BOLOS EN LA PROVINCIA DE LUGO}

En la provincia de Lugo se registra actualmente actividad bolística en 18 municipios, de los cuales 9 lo hacen con actividad deportiva federada (Xove, Ribadeo, Barreiros, Trabada, Riotorto, A Pontenova, A Pastoriza, Meira y Castro de Rei) y 9 con actividad deportiva no federada (Lourenzá, Mondoñedo, Cospeito, Navia de Suarna, Quiroga, Ribas de Sil, Sober, Pantón y Chantada) (figura 2).

Movimento, Porto Alegre, v. 20, n. 4, p. 1397-1421, out./dez. de 2014. 
Figura 2. Diferentes vías de entrada en Galicia a través del Camino de Santiago y municipios donde se registra actividad bolística.

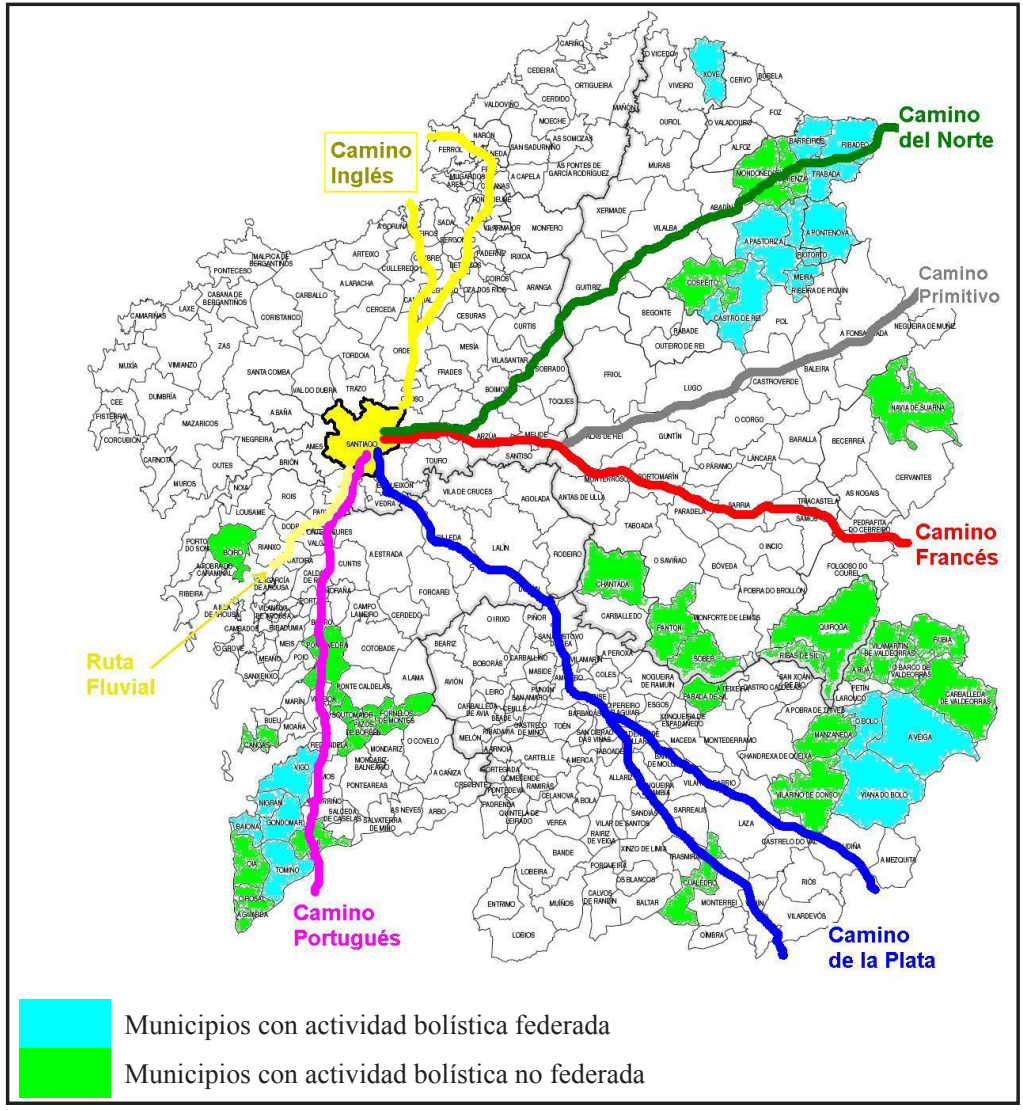

Fuente: elaboración propia

Nos encontramos con un total de 7 clubs deportivos federados de bolos en esta provincia, uno por municipio con actividad deportiva federada, a excepción del Club de Bolos Ribatra, que acoge jugadores de Ribadeo, Barreiros y Trabada (de ahí sus siglas) que participan en las competiciones organizadas por la Federación Gallega de Bolos en la modalidad de bolo tradicional (ver cuadro 1). 
Costumbres lúdicas en España: el juego de bolos ...

Cuadro 1. Relación de clubs deportivos federados de bolos de la provincia de Lugo.

\begin{tabular}{|c|c|}
\hline Municipio lugués & Club de Bolos \\
\hline Xove & Club Bolos Xove \\
\hline Castro de Rei & Club Bolos Cadaval \\
\hline Pastoriza & Club Bolos Campo Cruceiro \\
\hline Riotorto & Club Bolos Cruceiro de Meilán \\
\hline Meira & Club Bolos Ascasco de Orrea \\
\hline Ribadeo, Barreiros y Trabada & Club Bolos Ribatra \\
\hline Pontenova & Club Xogos Populares Tradicionais Santalla \\
\hline
\end{tabular}

Fuente: elaboración propia.

\subsection{El JUEGo de bOLOS en LA PROVINCIA DE OURENSE}

En la provincia de Ourense se registra actualmente actividad bolística en 12 municipios, de los cuales 3 lo hacen con actividad deportiva federada (A Veiga, O Bolo y Viana do Bolo) y 9 con actividad deportiva no federada (Parada de Sil, A Rúa, Vilamartín de Valdeorras, O Barco de Valdeorras, Rubiá, Carballeira de Valdeorras, Manzaneda, Vilariño de Conso y Cualedro) (ver figura 2).

De los tres municipios que tenemos referencia que se practica actividad deportiva federada de bolos, nos encontramos con un total de 5 clubs de bolos: uno en A Veiga, otro en O Bolo y tres en Viana do Bolo, municipio en el que el juego de bolos cobra mayor importancia y en el que se registra un mayor número de licencias federativas (ver cuadro 2).

Cuadro 2. Relación de clubs deportivos federados de bolos de la provincia de Ourense.

\begin{tabular}{|c|c|}
\hline Municipio ourensano & Club de Bolos \\
\hline \multirow{2}{*}{ Viana do Bolo } & Club Bolos Punxeiro \\
\cline { 2 - 2 } & Club Bolos Gonza \\
\cline { 2 - 2 } & Club Bolos Quintela \\
\hline O Bolo & Club Bolos O Bolo \\
\hline A Veiga & Club Bolos Baños \\
\hline
\end{tabular}

Fuente: elaboración propia.

Movimento, Porto Alegre, v. 20, n. 4, p. 1397-1421, out./dez. de 2014. 
6.3 El Juego de bolos en LA PROVINCIA de PonteVEdRA

En la provincia de Pontevedra se registra actualmente actividad bolística en 14 municipios, de los cuales 5 lo hacen con actividad deportiva federada (Tomiño, Gondomar, Baiona, Nigrán y Vigo) y 9 con actividad deportiva no federada (A Guarda, O Rosal, Oia, Tui, Pazos de Borbén, Fornelos de Montes, Soutomaior, Cangas y Pontevedra) (ver figura 2).

Nos encontramos con un total de 21 clubs deportivos federados de bolos en esta provincia (ver cuadro 3), la de mayor actividad de toda Galicia. Destaca en este aspecto la actividad registrada en los municipios de Baiona, Gondomar y Nigrán, con 7, 6 y 5 clubs de bolos respectivamente, siendo denominada esta zona de Val Miñor (comarca que debe su nombre al valle que forma el río Miñor y que pasa por estes tres municipios) la de mayor práctica de bolos en Galicia.

\subsection{El JUEGo de bolos EN LA PROVINCIA dE A CoRUÑA}

En la provincia de A Coruña únicamente se registra actividad bolística en un municipio (Boiro), como actividad deportiva no federada (ver figura 2). Actualmente están constituídos dos clubs de bolos (Club de Bolos de Pomardorrío y Asociación de Xogos e Deportes Tradicionais de Triñáns), de reciente creación (2013) y que forman parte de un proyecto de recuperación de este juego popular y tradicional en el municipio impulsado por el Servicio Municipal de Deportes de esta localidad. 
Costumbres lúdicas en España: el juego de bolos ...

Cuadro 3. Relación de clubs deportivos federados de bolos de la provincia de Pontevedra.

\begin{tabular}{|c|c|}
\hline Municipio pontevedrés & Club de Bolos \\
\hline \multirow{6}{*}{ Gondomar } & Club Bolos Peitieiros \\
\hline & Club Bolos San Vicente Mañufe \\
\hline & Club Bolos San Cibrán Donas \\
\hline & Club Bolos A Xunqueira \\
\hline & Club Bolos Concello de Gondomar \\
\hline & Club Bolos Sobreiro \\
\hline \multirow{5}{*}{ Nigrán } & Club Bolos Camos \\
\hline & Club Bolos do Ceán \\
\hline & Club Bolos da Carrasca \\
\hline & Club Bolos Santiago de Parada \\
\hline & Club Bolos San Roque \\
\hline \multirow{7}{*}{ Baiona } & Club Bolos Belesar-Urgal \\
\hline & Club Bolos Medialdea \\
\hline & Club Bolos A Rega \\
\hline & Club Bolos Estivada \\
\hline & Club Bolos Portameán \\
\hline & Club Bolos Morade \\
\hline & Club de Clásicos e Populares Val Miñor \\
\hline \multirow{2}{*}{ Tomiño } & Club Bolos Vilachán \\
\hline & Club Bolos Forcadela \\
\hline Vigo & Club Bolos Valladares San Bartolomé \\
\hline
\end{tabular}

Fuente: elaboración propia.

\section{Discusı́ón}

El Camino de Santiago es la vía más importante por la que se reciben influencias extranjeras y se adoptan costumbres de otros países. A partir del siglo IX los caminos de Galicia se llenaron de peregrinos procedentes sobre todo de Francia y Alemania, que penetraban en la península a través de los Pirineos y se dirigían a la

Movimento, Porto Alegre, v. 20, n. 4, p. 1397-1421, out./dez. de 2014. 
capital gallega a través de las vías del Camino Francés y del Camino del Norte preferentemente (RUIZ, 2000).

Partiendo de los trabajos de autores como Braun (1984), Ruiz (2000), Somorrostro (2001), Expósito (2006) o Pombo (2011) podemos comprobar que en Galicia entraba gente procedente de todos los lugares del mundo y por todos lados, siendo la principal la zona este de la comunidad gallega, que comunica con el resto de la península. Las influencias que se pudieron recibir de otros países, de otras culturas, sobre todo de la francesa o la germana, son de lo más variada posible y, aventurar que el juego de bolos es un legado que los peregrinos trajeron a Galicia no es desacertado, pues los principales pueblos en donde hoy en día se conserva este juego corresponden con lugares de paso de los diferentes Caminos de Santiago.

Los resultados expresados en la figura 2 nos indican que actualmente son tres las zonas principales de juego a la entrada de la comunidad gallega: al noreste (determinada por las influencias del Camino del Norte por la provincia de Lugo), al sureste (determinada por las influencias extranjeras que utilizaban la principal vía de acceso a Galicia a través del Camino Francés por la provincia de Ourense) y al suroeste (determinada por la entrada del Camino Portugués por la provincia de Pontevedra).

\subsection{El juego de bolos al final del Camino del Norte}

El Camino del Norte, también llamado Camino de la Costa, fue inicialmente el más utilizado para las peregrinaciones procedentes del Norte de Europa, recorriendo toda la costa cantábrica (País Vasco, Cantabria y Asturias) hasta llegar a Galicia, al pueblo de Ribadeo, en la provincia de Lugo. Se destaca un elevado nivel de práctica de bolos en esta zona, sobre todo a su entrada en la comunidad gallega, donde la modalidad de juego para la competición federada (unificada por la Federación Gallega de Bolos) es el bolo celta o tradicional pero, a nivel interno, se sigue jugando a la modalidad propia de cada zona, que en este caso son dos: los bolos ó canteiro

Movimento, Porto Alegre, v. 20, n. 4, p. 1397-1421, out./dez. de 2014. 
y los bolos á fila. Estamos de este modo ante una de las zonas de mayor práctica en toda Galicia (ver figura 2), que guarda una evidente conexión con el Camino del Norte, lugares que además del juego de bolos conservan la tradición de jugar en fiestas y celebraciones, así como nombres propios para referirse a lugares emblemáticos por donde pasa esta vía (SOMORROSTRO, 2001).

\subsection{El Juego de bolos al final del Camino Francés y el Camino de la Plata}

El Camino Francés, que se inicia en Roncesvalles, constituye la columna vertebral del Camino de Santiago (POMBO, 2011), denominándose Calle Mayor de Europa. Esta es la vía de mayor tránsito y las investigaciones nos indican que los lugares con influencia directa de este lugar de paso de los peregrinos registran un alto nivel de actividad bolística, aspecto en el que colabora la influencia del Camino de la Plata, otra vía que nace en Sevilla y termina en Astorga, enlazando con el Camino Francés, pero que a través de una variante abre una nueva vía de entrada en Galicia, paralela al Camino Francés, y entre los cuales se registra una de las zonas de Galicia con mayor actividad (ver figura 2).

Una parte de las referencias escritas sobre el juego de bolos en Galicia nos lleva a la cara este de la provincia de Ourense (VEGA, 1990), en la que destaca por encima de todos la actividad bolística en los municipios de O Bolo, A Veiga y Viana do Bolo (BERMÚDEZ, 2007). Sobre la modalidad de juego en esta zona, indicar que se trata de un juego de pasabolo, en el que se colocan 6 bolos cilíndricos en fila sobre una superficie de piedra para intentar derribarlos y desplazarlos lo más lejos posible a partir del lanzamiento de una bola esférica de aproximadamente $2 \mathrm{~kg}$ de peso. Esta forma de juego corresponde con la modalidad unificada por la Federación Gallega de Bolos, pero al igual que en los municipios de la provincia de Lugo (influenciados por el Camino del Norte), cada municipio sigue conservando su propia modalidad, practicada de igual forma o con mayor intensidad que la modalidad federada.

Movimento, Porto Alegre, v. 20, n. 4, p. 1397-1421, out./dez. de 2014. 
A veces, no se trata más que de variantes en el número de bolos, en su colocación, en el número de lanzamientos o en otros pequeños aspectos, pero que para cada localidad refleja la forma de juego de sus antepasados.

Pero la actividad federada no es la única que se refleja en esta zona, ya que en el valle de confluencia entre los Caminos del Norte y el Camino de la Plata se registra una fantástica actividad bolística, en el que 14 municipios ( 5 de la provincia de Lugo y 9 de la provincia de Ourense) practican el juego de bolos de forma autorregulada, donde las reglas son controladas por los propios jugadores y en la que estos son los gestores de su propia actividad lúdica.

\subsection{El Juego de bolos al final del Camino Portugués}

La peregrinación jacobea desde Portugal se intensifica a partir de la independencia de este país a mediados del siglo XII, aunque presumiblemente ya existía en la época altomedieval. Desde ese momento, el culto jacobeo y la peregrinación a Compostela, considerada como una de las señas de identidad de la cultura europea, tuvo en tierras lusitanas una proyección muy importante. Este camino empieza en Lisboa y llega a Tuy con un recorrido cercano a la costa, discurriendo del mismo modo hasta Santiago de Compostela, donde que enlaza con el camino procedente de la ruta fluvial. El discurrir de la práctica de los bolos en Galicia fue homogéneo con el paso del tiempo, empezando a diferenciarse a medida que nos acercamos a la actualidad, con el correspondiente bajón en el nivel de actividad. Pero esta tendencia de decaimiento consiguió estancarse en la zona sur de la provincia de Pontevedra en gran parte gracias a la actividad reglada proporcionada por la Federación Gallega de Bolos, que en la actualidad registra la inscrición de 21 clubs federados que acogen cerca de 300 licencias federativas. La modalidad propia de esta zona no difiere mucho de las existentes en las otras provincias gallegas en cuanto a su forma de juego (modalidad de pasabolo), aunque sí en el número de bolos empleados (18) y en su forma y tamaño (menos gruesos y más alargados).

Movimento, Porto Alegre, v. 20, n. 4, p. 1397-1421, out./dez. de 2014. 
7.4 El Camino Primitivo y las RUtas marítimas a través del Camino Inglés Y La Ruta FluVial.

El Camino Primitivo es considerado el primer camino jacobeo y se mantuvo durante todo el siglo IX pero, a partir del siglo $\mathrm{X}$ esta vía sufre un descenso importante en su afluencia al tiempo que se consolida el Camino Francés, aunque siguió canalizando el flujo de peregrinos procedentes del norte. En este caso, no observamos una influencia determinante de esta vía con los lugares donde actualmente se refleja un alto nivel de práctica, aunque en un pasado si pudo tener su importancia en el valle que formaría en conjunto con el Camino del Norte, principal zona de práctica al noreste de la comunidad gallega.

No observamos tampoco ningún tipo de influencia de las rutas marítimas a través del Camino Inglés y la Ruta Fluvial, aunque esta afirmación la realizamos en base a los datos que manejamos actualmente, siendo probable que en un pasado la situación fuera totalmente diferente.

Esto nos confirma que los caminos de entrada a Galicia se muestran como las zonas de mayor intercambio cultural, al establecerse en ellos unos vínculos de comunicación muy importantes entre los residentes y los peregrinos. Estas zonas son también etapas de descanso, donde el objetivo (llegar a Santiago de Compostela) está a dos o tres días de camino en el mejor de los casos; esto obliga a los peregrinos a pasar más tiempo en estas localidades, a relacionarse más con los vecinos, a compartir con ellos sus propias costumbres, a aprovechar el tiempo libre para disfrutar, para practicar actividades lúdicas y de diversión, donde seguramente el juego de bolos tendría su particular espacio al tratarse del divertimiento típico de la época.

A medida que nos acercamos a Santiago de Compostela, el nivel de práctica va decreciendo, del mismo modo que el interés del peregrino en realizar otras cosas que no sea ver la catedral y rendirse a la devoción religiosa que encierra el Camino. De hecho

Movimento, Porto Alegre, v. 20, n. 4, p. 1397-1421, out./dez. de 2014. 
no hay ninguna localidad a menos de 50 kilómetros de la capital donde se registre actividad bolística y, hasta los principales núcleos de práctica, se superan ampliamente los 100 kilómetros. Y a pesar de que no dejan de ser hipótesis, lo cierto es que las circunstancias, las referencias encontradas y los hechos, sobre todo los hechos, nos llevan a pensar en que así pudieron suceder las cosas.

A lo largo de este estudio podemos darnos cuenta de la importancia que supone para un determinado pueblo o comunidad la conservación de las tradiciones propias, historias donde se refleja la forma de ser de los antepasados y que nos muestran las raíces de procedencia. Supone una forma compleja de acercarnos a nuestra historia pero, al mismo tiempo, supone conocer y aceptar lo que somos, seguir siendo nosotros mismos sin negar el futuro y asumiendo el pasado (CABRAL, 1985).

\section{Conclusiones}

La temática acerca de los juegos populares y tradicionales es objeto de estudio cada día con mayor profusión entre los investigadores, enfocado principalmente a aspectos socioculturales y educativos. El juego de bolos, a pesar de la importancia que se le atribuye dentro de este colectivo de juegos populares y tradicionales, necesita de estudios de mayor profundidad para llegar a conocer la realidad de una de las actividades lúdicas más practicadas a lo largo de la historia. La escasez de publicaciones acerca del juego de bolos hace que los relatos orales cobren mayor importancia, siendo la principal fuente de conocimiento que nos podemos encontrar actualmente en España.

El Camino de Santiago constituye la principal ruta de peregrinación cristiana que tuvo una gran importancia a lo largo de la historia, dejando una impronta artística y cultural muy importante, jugando un papel fundamental en el fomento de intercambios culturales entre la Península Ibérica y el resto de Europa. A partir de los resultados obtenidos en la investigación, creemos que hay evidencias suficientes que nos indican que los

Movimento, Porto Alegre, v. 20, n. 4, p. 1397-1421, out./dez. de 2014. 
peregrinos que durante muchos años se acercaron a Galicia a través de las diferentes vías del Camino de Santiago dejaron en esta comunidad un importante legado cultural y artístico, dentro del cual podemos destacar el juego de bolos.

Los resultados ponen de manifiesto que en las zonas de Galicia donde se registra una práctica del juego de bolos más profusa e intensa se corresponde con los municipios gallegos situados en las principales vías de acceso a Galicia por el Camino de Santiago (Norte, Francés y Portugués), diluyéndose a medida que nos acercamos a la capital gallega. Aunque en la actualidad la zona de mayor práctica corresponde a la provincia de Pontevedra (relación con el camino portugués), en otros tiempos posiblemente esta circunstancia fuera semejante en las provincias de Lugo y Ourense (caminos del Norte y Francés), pues el tránsito de peregrinos hacia la capital gallega, más frecuente por unas vías que por otras, era intenso por todas ellas.

Se observan asimismo influencias extranjeras (sobre todo germanas y francesas) de los peregrinos en su camino a Santiago de Compostela, que aportaron sus tradiciones, costumbres y gustos, poniendo en contacto la comunidad gallega con las nuevas tendencias y las modas de los principales países europeos, entre ellos, el juego de bolos.

La importancia de conservar las tradiciones propias de cada pueblo supone conectarse con sus orígenes, con sus raíces, con sus antepasados. El juego de bolos forma parte de la historia como uno de las actividades lúdicas más practicadas, cuyo legado aún es visible en las cuatro provincias gallegas. 


\begin{abstract}
Playful customs in Spain: the game of the skittles in the final stage of the Way of Santiago

Abstract: The study sought to identify and analyze the playful practices of residents of northwestern Spain and their relation to the distinct sea-land routes of the Way of Santiago that enter Galicia. Data were obtained through a qualitative methodology, using semistructured interview and literature review. A relevant relationship was found between the routes to enter the Way of Santiago in Galicia and the towns where the game of skittles is still played, indicating the influence of pilgrims on their way to Santiago with spiritual ends, who transfer their customs and traditions.

Key words: Culture. Play and playthings. Leisure activities.
\end{abstract}

\title{
Hábitos de lazer em Espanha: o jogo de bolos na fase final do caminho de Santiago \\ Resumo: $O$ estudo procurou identificar e analisar as práticas lúdicas dos habitantes do noroeste da Espanha e a relação que se estabelece com as diferentes vias marítimo-terrestres do Caminho de Santiago que penetram a Galícia. Os dados foram obtidos através de uma metodologia qualitativa, utilizando a entrevista semiestruturada e a revisão da literatura. Constatou-se um grande paralelo entre rotas de entrada do Caminho de Santiago na Galícia e os municípios onde atualmente ainda se joga bolos, indicando as influências de peregrinos no seu caminho a Santiago para fins espirituais, transferindo seus costumes e tradições. \\ Palavras-chave: Cultura. Jogos e brinquedos. Atividades de lazer.
}

\section{REFERENCIAS}

BARROSO, Concepción. Las bases sociales de la ludopatía. 2003. 403f. Tesis (doctorado) - Departamento de Sociología. Universidad de Granada, Granada, 2003.

BAUTISTA, José Manuel. El juego como método didáctico: propuestas didácticas y organizativas. Granada: Adhara, 2002.

BERMÚDEZ, María. Los bolos en Galicia. Lugo: Lulu.com, 2007.

BRAUN, Julio. Bolos y cultura. Santander: Artes Gráficas Resma, 1984.

BUZÁN, Tony. Tu mente en forma. Barcelona: Urano, 2004.

CABRAL, António. Jogos populares portugueses. Porto: Domingos Barreira, 1985.

CAGIGAL, José María. Obras selectas. Cádiz: COE, 1996.

Movimento, Porto Alegre, v. 20, n. 4, p. 1397-1421, out./dez. de 2014. 
Costumbres lúdicas en España: el juego de bolos ...

CANALES, Manuel. Metodologías de investigación social: Introducción a los oficios. Santiago de Chile: LOM, 2006.

COOMBS, Philip. La crisis mundial en la educación: Perspectivas actuales. Madrid: Santillana, 1985.

CORBETTA, Piergiorgio. Metodología y técnicas de investigación social. Madrid: McGraw-Hill, 2007.

CORTIZAS, Antón. Chirlosmirlos: Enciclopedia dos xogos populares. Vigo: Xerais, 2001.

COSTAS, Fernando Javier; RODRÍGUEZ, José Manuel. Os xogos de birlos no Suroeste de Galicia (Val Miñor e Baixo Miño). Boletín do Instituto de Estudos Vigueses, Vigo, v. 6, n .6, p.147-174, ene./dic. 2000.

DONCEL, Lucio. Deportes tradicionales de fuerza en España. Madrid: Visión Libros, 2010.

ECHEVARRÍA, Alberto. Los Caminos de Santiago. Madrid: Gondo, 2008.

EXPÓSITO, Juan. El juego y deporte popular, tradicional y autóctono en la escuela. Los bolos huertanos y bolos cartageneros. Sevilla: Wanceulen, 2006.

FERNÁNDEZ, Álvaro. Los bolos en España. Gijón: Baukunión, 1978.

FLICK, Uwe. Introducción a la investigación cualitativa. Madrid: Morata, 2007.

HERNÁNDEZ, José. Catálogo de los deportes y juegos tradicionales canarios de adultos: Estudio praxiológico, institucional y documental. Barcelona: Inde, 2007.

HUIZINGA, Johan. Homo ludens. Madrid: Alianza, 1972.

LARA, Amador. Transmission of social and educational values through sport. Journal of Sport and Health Research, Jaén (España), v.3. n. 1, p. 5-6, jan./apr. 2011.

LAVEGA, Pere. El juego: teorías y características del mismo. El juego como actividad física organizada. Estrategias del juego. En: Temario desarrollado de los contenidos específicos del área de Educación Física para el acceso al Cuerpo de Profesores de Enseñanza Secundaria. Barcelona: Inde, 1994. p. 4168.

LAVEGA, Pere. Del joc a l'esport. Estudi de bitlles al Pla d'Urgell (Lleida). 1996. 1337 p. Tesis (doctorado) - Departament de Teoria i Història de l'Educació, Universitat de Barcelona; Barcelona, 1996.

LAVEGA, Pere. Aplicación educativa/recreativa del juego a partir de sus distintos niveles de organización. En: WORLD PLAY CONFERENCE "PLAY AND SOCIETY". 20th., Comunicación presentada en el 20th, ICCP Facultad Motricidad Humana. Lisboa, 1997.

Movimento, Porto Alegre, v. 20, n. 4, p. 1397-1421, out./dez. de 2014. 
LAVEGA, Pere. Juegos y deportes populares-tradicionales. Barcelona: Inde, 2000a.

LAVEGA, Pere. La riqueza motriz y cultural de los juegos de los bolos. En: CONGRESO NACIONAL DE BOLOS, 1. Libro de actas. Santander: Federación Cántabra de Bolos, 2000b.

LAVEGA, Pere. Los juegos y deportes tradicionales en Europa: entre la tradición y la modernidad. Revista APUNTS de Educación Física y Deportes, Barcelona, n. 85, p. 68-81, jul./sept. 2006.

LIÑARES, Amancio. Ramón Martínez López. Santiago de Compostela: TesCtres, 2007.

LÓPEZ, Juan Luis. Los juegos tradicionales de lanzamiento y precisión en el aula de Educación Física. Una aplicación práctica. Revista Digital Educación Física y Deportes, Buenos Aires, n. 136, p. 1, 2009. Disponible en <http://www.efdeportes. com/efd136/los-juegos-tradicionales-de-lanzamiento.htm>. Acceso: 12 feb. 2012.

MELERO, Mario. Aproximación científica a los juegos populares, tradicionales y autóctonos de España. Revista digital Educación Física y Deportes, Buenos Aires, n. 133, p. 1, 2009. Disponible en <http://www.efdeportes.com/efd133/juegospopulares-tradicionales-y-autoctonos.htm>. Acceso: 12 feb. 2012.

MORENO, Cristóbal. Juegos y deportes tradicionales en España. Madrid: Alianza, 1992.

MUÑOZ, Juan Manuel. Los mapas mentales como técnica para integrar y potenciar el aprendizaje holístico en la formación inicial de maestros/as. 2009. 415 p. Tesis (doctorado) - Facultad de Ciencias de la Educación Córdoba: Universidad de Córdoba, Córdoba, 2009.

NAVARRO, Vicente. El afán de jugar. Barcelona: Inde, 2003.

ONTORIA, Antonio; GÓMEZ, Juan Pedro; LUQUE, Ángela. Aprender con mapas mentales. Madrid: Narcea, 2006.

PARLEBÁS, Pierre. Elementos de sociología del deporte. Málaga: Unisport, 2003.

PAZOS, José María. La gestión del deporte en la administración pública local en clave educativa de motricidad humana. Un estudio de caso: Porto do Son. 2010. 412 p. Tesis (doctorado) - Universidad de Santiago de Compostela, Santiago de Compostela, 2010.

PÉREZ, Ricardo; TABERNERO, Alberto. Juegos populares en Galicia. Santiago: Lea, 1997.

POMBO, Antón. El Camino de Santiago en tu mochila. Madrid: Anaya, 2011.

RODRÍGUEZ, José Eugenio. O estudo do xogo dos bolos en Boiro: aspectos históricos, estado actual de práctica e perspectivas de futuro. 2013. 494 p. Tesis (doctorado) - Departamento de Educación Física e Deportiva, Universidad de A Coruña, 2013.

Movimento, Porto Alegre, v. 20, n. 4, p. 1397-1421, out./dez. de 2014. 
ROMANÍ, Arturo. Xogos infantiles de Galicia. Santiago: Follas Novas, 1979.

ROMERO, Juan Bautista. El juego deportivo y musical como transmisor de valores no violentos. Revista Wanceulen Educación Física Digital, n. 3, p. 1-10, 2007. Disponible en <http://rabida.uhu.es/dspace/bitstream/handle/10272/1707/b1534966. pdf?sequence=1>. Acceso: 26 feb. 2012.

RUIZ, Guillermo; CABRERA, Dolores. Los valores en el deporte. Revista de educación, Madrid, n. 335, p. 9-19, sept./dic. 2004.

RUIZ, José Gerardo. Estudios de los bolos en Asturias: aspectos históricoculturales, modalidades, elementos y materiales de juego. Estado actual de su práctica. 2000. 455 f. Tesis (doctorado) - Departamento de Educación Física y Deportiva, Universidad de Granada, Granada, 2000.

SANTOS, Xosé Manuel. El Camino de Santiago: turistas y peregrinos hacia Compostela. Cuadernos de Turismo, Murcia, n. 18, p. 135-150, jul/dic. 2006.

SOMORROSTRO, José. Los bolos de Santa Comba de Orrea (Riotorto, Lugo). Recuperación de un juego popular. Revista Lucensia. Miscelánea de Cultura e Investigación, Lugo, n. 22, p. 143-152, ene./jun. 2001.

TÓJAR, Juan Carlos. Investigación cualitativa. Comprender y actuar. Madrid: La Muralla, 2006.

VEGA, Tomás. Dos enredos tradicionales de la comarca Navea-Bibei (Orense). Boletín de Estudios del Seminario "Fontán Sarmiento" de Hagiografría, Toponimia y Onomástica de Galicia, Santiago de Compostela, n. 11, p. 60-65, ene./dic. 1990.

VEIGA, Francisco. Xogo popular galego e educación. Vixencia educativa e función de identificación cultural dos xogos e enredos tradicionais. 1998. n. páginas. Tesis (doctorado). Facultade de Ciencias da Educación, Santiago de Compostela: Universidad de Santiago de Compostela, 1998.

VIGNE, Mickaël. Las actividades tradicionales de ocio como reflejo de una sociedad. Revista Científica Digital Acción Motriz, Las Palmas de Gran Canaria (España), n. 7, p. 62-76, jul./dic. 2011. Disponible en <http://www.accionmotriz.com/revistas/7/7 6. pdf>. Acceso: 12 feb. 2012.

Endereço para correspondência:

José Eugenio Rodríguez Fernández

C/ Corviño n 72. Cabo de Cruz, 15939.

Boiro. A Coruña. España.

Recebido em: 02.04.2014

Aprovado em: 21.09.2014

Movimento, Porto Alegre, v. 20, n. 4, p. 1397-1421, out./dez. de 2014. 